\title{
Mantillo de las especies leñosas de matorRales del NO De la Patagonia: abundancia, COMPOSICIÓN, Estructura Y HETEROGENEIDAD
}

\author{
MANUEL DE PAZ ${ }^{1}$, MIRIAM E. GOBBI ${ }^{2}$ y ESTELA RAFFAELE ${ }^{1}$
}

\begin{abstract}
Summary: Litter of woody species of NW Patagonian shrublands: composition, structure and heterogeneity. Litter is an important component of ecosystems. It influences nutrient dynamics and microsite conditions (e.g., solar radiation, soil temperature and water retention). Consequently, litter could affect plant regeneration, either positively or negatively. Our aim was to study abundance, composition, heterogeneity and annual production of the litter produced by fifteen common woody species in shrublands of NW Patagonia. Total litter varied from 347.9 to $2534.7 \mathrm{gm}^{-2}$. Litter accumulation in these shrublands was lower than in Andean forests but similar to other shrublands of other regions. Also, annual production of litter was lower than in other ecosystems and similar to the Patagonian Monte. Litter heterogeneity was similar among species. Perennial species accumulated and produced more litter per year than deciduous species. These results could be important to understand the regeneration process and nutrient cycling at the community scale. In both functional groups we found some species with extreme values in most variables and others which intermediate values which represent a variation gradient. This provides this system with a high diversity of microsites associated with litter.
\end{abstract}

Key words: Senescent matter, leaf litter, deciduous, evergreen.

\begin{abstract}
Resumen: El mantillo es un componente importante de los ecosistemas, que afecta tanto la dinámica de nutrientes como las condiciones de micrositio (e.g., radiación, temperatura y retención de humedad del suelo) y, en consecuencia, puede tener diferentes tipos de efectos sobre la regeneración de las especies. El objetivo de este trabajo fue estudiar, bajo el dosel de 15 especies leñosas, muy frecuentes en los matorrales del NO de la Patagonia, la abundancia, composición, estructura y heterogeneidad del mantillo y la producción de material senescente/año. El mantillo total acumulado varió entre 347,9 y $2534,7 \mathrm{gm}$ 2. El mantillo y la producción de material senescente/año fue baja comparada con otros ecosistemas y similar a la del monte Patagónico. La heterogeneidad del mantillo fue similar entre la mayoría de las especies. Las especies perennifolias tuvieron mayor acumulación de mantillo y producción de material senescente por año que las especies caducifolias. Esto podría tener implicancias en la regeneración y ciclado de nutrientes a escala de comunidad. En ambos grupos funcionales se encontraron especies con valores extremos y otras que representan un gradiente de variación. Esto contribuye a darle a este sistema una alta diversidad de micrositios asociada al mantillo
\end{abstract}

Palabras clave: Material senescente, hojarasca, caducifolias, perennifolias.

\section{INTRODUCCIÓN}

El mantillo es un componente importante de los ecosistemas, porque regula el ciclo de nutrientes

\footnotetext{
${ }^{1}$ Laboratorio Ecotono, INIBIOMA (CONICET-Universidad Nacional del Comahue).

2 Depto. de Biología, CRUB, Universidad Nacional del Comahue e INIBIOMA (CONICET-Universidad Nacional del Comahue).
}

e influye sobre las características ambientales de los micrositios donde se acumula. Se considera mantillo a la sumatoria de hojarasca (hojas senescentes caídas), ramas finas, flores, frutos y materia orgánica particulada depositados sobre el suelo (Del Valle-Arango, 2003; Carmona et al., 2006; Pérez et al., 2006; Varela et al., 2006). Los efectos más importantes del mantillo sobre las variables microambientales son la disminución de la temperatura y la radiación durante el verano, la conservación de la humedad del suelo durante 
mayor tiempo y la amortiguación del efecto de las heladas, las lluvias y el viento (Facelli \& Pickett, 1991b; Facelli et al., 1999; Gutiérrez \& Squeo 2004). Este estrato constituye el ambiente donde se desarrolla gran parte de la micro y meso biota del suelo y, por lo tanto, tiene fuertes implicancias en la conservación de la biodiversidad de estos grupos. La abundancia, composición, y las características del mantillo dependen de la tasa de producción, la composición química y las propiedades físicas de las hojas senescentes de las mismas, el aporte de otro tipo de material vegetal (ramas, corteza, frutos, etc.) y las tasas de descomposición. Estos factores a su vez dependen de las especies que producen el material, de la comunidad microbiana y de las condiciones ambientales (Facelli \& Facelli, 1993; Vivanco \& Austin, 2006; Vivanco \& Austin, 2008).

Las características físicas, tales como la dureza, área foliar específica (AFE), peso y área foliar, el total del mantillo producido y el tipo de materiales que lo componen, conforman la estructura de la hojarasca. Dicha estructura afecta de manera directa sobre la descomposición y, por lo tanto, la cantidad de mantillo que se acumula bajo el dosel de las plantas. Algunas de las características mencionadas están asociadas entre sí, por ejemplo el AFE se correlaciona negativamente con la dureza y positivamente con la velocidad de descomposición. (Pérez-Harguindeguy et al., 2000). La heterogeneidad del mantillo representa la diversidad de materiales que lo componen, y puede estar dada tanto por el aporte de más de una especie, como por diferencias en la tasa de descomposición de alguno de los componentes del mantillo. Esta heterogeneidad puede influir en la microbiota del suelo y las condiciones de micrositios, producir interacciones entre los diferentes materiales o afectar la calidad promedio de los materiales en descomposición y así afectar las tasas de acumulación y descomposición del mantillo (Hansen \& Coleman, 1998; Pérez Harguindeguy et al., 2008). Por ejemplo, un mantillo más heterogéneo podría captar mayor cantidad y diversidad de materiales al contar con más diversidad de intersticios que un mantillo más homogéneo. Además, de estos factores, las tasas de descomposición tienen relación inversa con el tamaño del material en el suelo, y consecuentemente, la descomposición será más veloz en el mantillo con partículas de menor tamaño (Barrera et al.,
2004). Además, los órganos de vida corta, como hojas y ramas finas, usualmente tienen menos lignina y compuestos secundarios (Coley et al., 1985; Berendse et al., 1987) y consecuentemente se descomponen más rápidamente (Rhoades \& Cates, 1976; Coley et al., 1985; Choundhury, 1988). En contraste, los órganos de larga vida, como las ramas y troncos, tardan más en descomponerse debido al mayor contenido de lignina y compuestos secundarios (Fenny, 1970). Además, la acumulación de estos materiales se relaciona con la disposición espacial, la arquitectura de las especies, factores ambientales (Aerts, 1997), y con la estructura del mantillo que puede afectar la captación de material senescente.

El mantillo puede condicionar negativamente la regeneración de la vegetación inhibiendo la germinación, actuando como barrera física para el desarrollo de la radícula, dificultando el establecimiento de plántulas (Baskin \& Baskin, 1989; Facelli \& Pickett, 1991c; Facelli \& Pickett, 1991a; Xiong et al., 2001), y también incrementando la depredación de plántulas por un aumento en la abundancia de herbívoros (Facelli, 1994). Además, algunas especies producen hojarasca con las sustancias alelopáticas que pueden dañar total o parcialmente, la germinación y el crecimiento de las plantas (Larcher, 2001). En otro sentido, el mantillo condiciona la cantidad y calidad de luz recibida, reduce el efecto de las heladas (Baskin \& Baskin, 1989; Facelli \& Pickett, 1991c; Gutiérrez \& Squeo 2004). Además, el mantillo condiciona la cantidad y calidad de luz recibida, y el sentido de este efecto depende de los requerimientos de las plántulas. Estos efectos antagónicos se relacionan tanto a la heterogeneidad del mantillo intra y entre especies, como con los requerimientos en la regeneración y crecimiento de las especies, y muchos de los mecanismos involucrados son aún desconocidos (Aguiar et al., 1992; Cavieres et al., 2008).

En Patagonia existen grandes áreas de matorrales que han sido sometidos reiteradamente a incendios y ganadería y que conforman, particularmente luego de fuegos relativamente recientes, ambientes de alta heterogeneidad, con parches de vegetación intercalados entre superficies importantes de suelo desnudo (Cavallero, 2012). La mayoría de las especies de estos matorrales son claves para la regeneración y mantenimiento tanto de los matorrales, como de los bosques secos y 


\section{M. del Paz et al. - Mantillo de especies leñosas de matorrales de la Patagonia}

mixtos. Estas especies, algunas de ellas citadas además como nodrizas (Raffaele \& Veblen, 1998; Kitzberger et al., 2000; Nuñez et al., 2009), sirven como perchas o posaderos para las aves, siendo muy importantes en la dispersión de especies vegetales (Cavallero, 2012). Bajo este contexto es esperable que estas "islas de vegetación de especies leñosas", además de favorecer el establecimiento y supervivencia de otras especies, de producir sombra y retener humedad en el suelo, aumenten la fertilidad del suelo mediante el aporte del mantillo (Gutiérrez \& Squeo 2004).

El objetivo de este trabajo fue estudiar, bajo el dosel de 15 especies leñosas, muy frecuentes en los matorrales del NO de la Patagonia, la abundancia, composición y heterogeneidad del material senescente producido por año y el mantillo. Asimismo se analizó si existen diferencias de los distintos componentes del mantillo entre las especies y/o entre grupos de especies (caducifolias y perennifolias). Este tipo de información es permitirá comprender los posibles efectos del mantillo (e.g. barrera física para la germinación, retención de humedad, sustancias alelopáticas) de estas especies sobre la germinación de plántulas, descomposición de la materia orgánica y dinámica de nutrientes.

En este estudio se propone como hipótesis general que los distintos grupos funcionales de arbustos (perennifolias vs. caducifolias) difieren en el aporte del material senescente y en las características del mantillo. Se espera que el material senescente proveniente de especies perennifolias sea menos abundante, con características asociadas comúnmente a bajas tasas de descomposición y dará origen a un mantillo más heterogéneo respecto del proveniente de especies caducifolias.

\section{MÉTodos}

Área de estudio

El estudio se realizó en la zona de transición estepa-bosque (ecotono) del NO de la Patagonia $\left(40^{\circ} 51^{\prime} 00^{\prime \prime}\right.$ a $41^{\circ} 11^{\prime} 58^{\prime \prime} \mathrm{S}$ y $71^{\circ} 3 ' 58,09^{\prime \prime}$ a $\left.71^{\circ} 26^{\prime} 13,09^{\prime \prime} \mathrm{O}\right)$. En esta zona la vegetación predominante está constituida por matorrales, tanto mixtos como dominados por Nothofagus antarctica (G.Forst.) Oerst., y bosques xéricos de Austrocedrus chilensis (D.Don) Pic.Serm.
\& Bizzarri. Típicamente, estos matorrales se encuentran dominados principalmente por numerosas especies rebrotantes como $N$. antarctica, Berberis microphylla G. Forst., Ribes cucullatum Hook. \& Arn., Schinus patagonicus (Phil.) I. M. Jonnst. y Diostea juncea (Giles \& Hook.) Miers y son ambientes más propensos a incendiarse comparado con los bosques (Veblen et al., 2011). Las especies dominantes mencionadas son capaces de rebrotar inmediatamente luego de un incendio, proporcionando las condiciones adecuadas para facilitar con el tiempo la recuperación de los bosques, siendo así especies claves en la sucesión post-fuego (Raffaele \& Veblen, 1998; Kitzberger et al., 2000; Nuñez et al., 2009).

Las precipitaciones son abundantes (ca. 1000 $\mathrm{mm}$ anuales), concentradas durante el invierno y escasas durante el período de máximo crecimiento de las plantas (verano). La temperatura media anual es de $6,3{ }^{\circ} \mathrm{C}$ y los vientos predominantes son del SO (Datos climáticos suministrados por EEAINTA Bariloche). Los suelos de la Región AndinoPatagónica son mayoritariamente derivados de cenizas volcánicas (Andisoles) y se caracterizan por una adecuada provisión de nutrientes, alta concentración y estabilidad de la materia orgánica y alta capacidad de almacenamiento de agua (Colmet-Daage et al., 1993; Colmet-Daage et al., 1995). El principal factor limitante de estos suelos es la baja disponibilidad de fósforo, que es retenido fuertemente en las arcillas alofánicas, pero la vegetación nativa se encuentra adaptada, a través de asociaciones micorrízicas (Carrillo et al., 1992; Fontenla et al., 1998). Estos suelos poseen buena estabilidad, con una fuerte tendencia a restablecer sus propiedades luego de disturbios (Gobbi, 2000). Sin embargo, los incendios disminuyen la cantidad de $\mathrm{N}$ y $\mathrm{C}$, la retención de agua en el suelo y la mineralización del $\mathrm{N}$, así como también provocan un aumento en la disponibilidad de $\mathrm{P}$ a mediano plazo y pérdidas importantes del mismo a largo plazo (Alauzis et al., 2004; Kitzberger et al., 2005; Paritsis et al., 2006).

Los matorrales estudiados se encuentran en zonas de contacto con diferentes tipos de bosques (e.g., Nothofagus dombeyii (Mirb.) Oerst., A. chilensis) o con la estepa. Se establecieron luego de incendios de entre 10 y 12 años (Veblen et al., 2003) y han sufrido extracciones de leña y tienen presión de herbívoros exóticos, principalmente 
liebre y, eventualmente, ganado vacuno y equino. Su ubicación y fisonomía, los convierte en sistemas altamente perturbados por el hombre. Sin embargo, son sistemas con alta diversidad de especies (Raffaele \& Veblen, 2001). En estos matorrales, las especies caducifolias poseen una cobertura similar a las especies perennifolias $(48,2 \%$ y $42,8 \%$ respectivamente, datos sin publicar, Tabla 1).

Los individuos de las especies estudiadas fueron seleccionados en matorrales ubicados en el ecotono estepa-bosque de igual edad post fuego y similares características ambientales respecto al tipo de suelo, régimen de precipitaciones y condiciones topográficas (e.g. pendiente, exposición).

\section{Diseño experimental}

Se seleccionaron 15 especies leñosas de alta representatividad en la zona. En la Tabla

Tabla 1. Especies de estudio y sus características foliares, abundancia y tamaño promedio ( $\pm E R)$ :

Duración de hojas en la planta, Tamaño y dureza de las hojas, Frecuencia en sitio de estudio $(F)$, Altura

en $\mathrm{m}(\mathrm{A})$, Área de dosel en $\mathrm{m}^{2}$ (D). Se llama hojas chicas a hojas verdes menores a $80 \mathrm{~mm}^{2}$, medianas

entre 81 y $300 \mathrm{~mm}^{2}$, grandes mayores a $3 \mathrm{~mm}^{2}$. Se llama hojas duras a las hojas verdes con dureza menor a $241 \mathrm{gmm}^{-2}$ y blandas a las hojas verdes mayor a $240 \mathrm{gmm}^{-2}$ (de Paz, datos sin publicar)

\begin{tabular}{|c|c|c|c|c|c|c|c|}
\hline Especie & Familia & $\begin{array}{l}\text { Duración } \\
\text { de hojas en } \\
\text { la planta }\end{array}$ & $\begin{array}{c}\text { Tamaño y dureza } \\
\text { de hojas }\end{array}$ & $\begin{array}{l}\text { Cobertura } \\
\text { media (\%) }\end{array}$ & $\mathbf{F}$ & A & D \\
\hline $\begin{array}{l}\text { Diostea juncea (Giles } \\
\& \text { Hook.) Miers }\end{array}$ & Verbenaceae & caducifolia $^{\text {a }}$ & Chicas y blandas & $5,2 \pm 1,5$ & 0,2 & $2,7 \pm 0,1$ & $5,8 \pm 0,8$ \\
\hline $\begin{array}{l}\text { Discaria articulata } \\
\text { (Phil.) Miers }\end{array}$ & Rhamnaceae & caducifolia & Chicas y blandas & $12,0 \pm 2,9$ & 0,4 & $0,6 \pm 0,1$ & $2,2 \pm 0,4$ \\
\hline $\begin{array}{l}\text { Discaria chacaye } \\
\text { (G. Dom.) Tortosa }\end{array}$ & Rhamnaceae & caducifolia & Chicas y blandas & $3,7 \pm 2,9$ & 0,3 & $1,7 \pm 0,1$ & $3,2 \pm 0,6$ \\
\hline $\begin{array}{l}\text { Escallonia virgata } \\
\text { (Ruiz \& Pav) Pers }\end{array}$ & Saxifragaceae & caducifolia & Chicas y blandas & $0,4 \pm 0,2$ & 0,1 & $2,1 \pm 0,1$ & $5,1 \pm 1,1$ \\
\hline $\begin{array}{l}\text { Nothofagus antarctica } \\
\text { (G.Forst.) Oerst. }\end{array}$ & Nothofagaceae & caducifolia & Medianas y blandas & $20,4 \pm 2,7$ & 0,5 & $2,4 \pm 0,2$ & $7,9 \pm 1,2$ \\
\hline $\begin{array}{l}\text { Ribes cucullatum } \\
\text { Hook. \& Arn. }\end{array}$ & Saxifragaceae & caducifolia & Chicas y blandas & $0,3 \pm 0,1$ & 0,2 & $1,2 \pm 0,1$ & $1,8 \pm 0,3$ \\
\hline Rosa rubiginosa ${ }^{\mathrm{b}} \mathrm{L}$. & Rosaceae & caducifolia & Grandes y blandas & $6,6 \pm 2,7$ & 0,3 & $1,7 \pm 0,2$ & $4,2 \pm 1,1$ \\
\hline $\begin{array}{l}\text { Acaena integerrima } \\
\text { Gillies ex Hook. \& Arn. }\end{array}$ & Rosaceae & perennifolia & Grandes y blandas & $6,2 \pm 1,9$ & 0,6 & $0,1 \pm 0,0$ & $1,5 \pm 0,6$ \\
\hline $\begin{array}{l}\text { Adesmia boronioides } \\
\text { Hook. f. }\end{array}$ & Fabaceae & perennifolia & Medianas y duras & $1,2 \pm 0,9$ & 0,1 & $1,0 \pm 0,1$ & $1,8 \pm 0,4$ \\
\hline $\begin{array}{l}\text { Baccharis obovata } \\
\text { Hook \& Arn. }\end{array}$ & Asteraceae & perennifolia & Chicas y duras & $0,4 \pm 0,2$ & 0,1 & $1,6 \pm 0,1$ & $3,5 \pm 0,7$ \\
\hline $\begin{array}{l}\text { Berberis microphylla } \\
\text { (Phil.) Miers }\end{array}$ & Berberidaceae & perennifolia & Chicas y duras & $4,9 \pm 1,3$ & 0,6 & $1,3 \pm 0,1$ & $3,4 \pm 1,2$ \\
\hline $\begin{array}{l}\text { Embothrium coccineum } \\
\text { J. R. Forts. \& G. Forts. }\end{array}$ & Proteaceae & perennifolia & Grandes y blandas & $0,8 \pm 0,3$ & 0,2 & $1,9 \pm 0,2$ & $3,5 \pm 0,9$ \\
\hline $\begin{array}{l}\text { Lomatia hirsuta (Lam) } \\
\text { Diels ex J.F.Macbr. }\end{array}$ & Proteaceae & perennifolia & Grandes y duras & $13,7 \pm 2,7$ & 0,3 & $2,9 \pm 0,4$ & $6,6 \pm 1,2$ \\
\hline Maytenus boaria Molina & Celastraceae & perennifolia & Medianas y duras & $3,4 \pm 1,4$ & 0,3 & $1,9 \pm 0,2$ & $2,1 \pm 0,5$ \\
\hline $\begin{array}{l}\text { Schinus patagonicus } \\
\text { (Phil.) I. M. Jonnst. }\end{array}$ & Anacardiaceae & perennifolia & Medianas y duras & $11,8 \pm 2,6$ & 0,6 & $2,1 \pm 0,1$ & $6,1 \pm 0,6$ \\
\hline
\end{tabular}

Características morfológicas de las especies tomadas de Correa (1976). Excepto la referencia de tamaño y dureza de las hojas definidos por datos propios de hojas verdes sin publicar. ${ }^{a}$ anatómicamente se la considera perennifolia porque retiene la vaina alrededor del tallo, pero pierde la lámina de la hoja durante el invierno. ${ }^{\mathrm{b}}$ Exótica. 


\section{M. del Paz et al. - Mantillo de especies leñosas de matorrales de la Patagonia}

1 se mencionan las especies y sus principales características morfológicas. Para cada especie se estimó su cobertura porcentual promedio y frecuencia con el método de los cuadrantes en el sitio de estudio (Mostacedo \& Fredericksen, 2000) en 60 parcelas de $4 \mathrm{~m}^{2}$ cada una. Además, se eligieron aleatoriamente 10 individuos de similar altura y tamaño de dosel para cada especie. En cada individuo se registró la altura y el área de dosel, este último a partir del diámetro mayor, el perpendicular a este y la forma del dosel (circular, rectangular, cuadrangular, Tabla 1). Para caracterizar el mantillo se midieron las siguientes variables:

- La profundidad se midió en 5 puntos bajo el dosel de cada individuo. Utilizando una regla se registró la distancia entre la superficie del suelo y la superficie del mantillo.

- La abundancia, se colectó el mantillo en una superficie de $40 \mathrm{~cm}^{2}$ bajo el dosel de cada planta. En laboratorio se separó manualmente el material en los siguientes componentes: hojarasca (hojas senescentes propias de la especie), ramas finas ( $<1 \mathrm{~cm}$ de diámetro), hierbas y pastos (gramíneas y graminoides), materia orgánica irreconocible mayor a $1 \mathrm{~mm}(\mathrm{MOI}>1 \mathrm{~mm})$ y MOI menor a 1 $\mathrm{mm}(\mathrm{MOI}<1 \mathrm{~mm})$. En el caso de $A$. integerrima las hojas y ramas senescentes quedan adheridas a la base de las ramas vivas por largo tiempo, por lo cual debieron ser extraídas manualmente. Las últimas dos categorías fueron separadas con el uso de un tamiz. Posteriormente se secó a $60{ }^{\circ} \mathrm{C}$ por 72 hs. y se registró el peso seco.

- La caída anual de material senescente se estimó en las diez especies más abundantes (ver Tabla 1). Se colectó hojarasca propia de la especie, hojarasca de hierbas, pastos y otras especies arbustivas, ramas finas $(<1 \mathrm{~cm}$ de diámetro) y frutos en trampas de $40 \mathrm{~cm}^{2}$, con una base de malla plástica de $1 \mathrm{~mm}$. Se seleccionaron cinco individuos por especie y se colocó una trampa bajo el dosel de cada uno. En el caso de $A$. integerrima se marcó la parte de la planta donde previamente se había retirado el mantillo, de manera que toda parte senescente encontrada en ese sector se pudiera atribuir al año en curso. Las trampas se colocaron a fines de invierno del año 2009 y se retiraron a fines del otoño de 2010. Cada quince días las trampas fueron revisadas, el material vegetal retirado y en laboratorio se cuantificó el peso seco de hojarasca, ramas y de frutos $\left(60^{\circ} \mathrm{C}\right.$ por $\left.72 \mathrm{hs}\right)$. La caída anual se calculó como la sumatoria del material cuantificado en todas las fechas estimado por metro cuadrado.

- El área foliar y peso foliar seco $\left(60^{\circ} \mathrm{C}\right.$ por $\left.72 \mathrm{hs}\right) \mathrm{de}$ las hojas senescentes se determinó en 15 hojas de cada individuo, elegidas aleatoriamente. El área foliar se determinó siguiendo la metodología propuesta por Cornelissen et al. (2003). En el caso de las hojas compuestas se consideraron los folíolos más el raquis. El área foliar específica (AFE) para cada hoja se estimó como área foliar/ peso foliar $\left(\mathrm{mm}^{2} \mathrm{~g}^{-1}\right)$.

- La dureza de las hojas senescentes se determinó en 30 hojas por individuo, elegidas al azar y recientemente depositadas sobre el suelo. Se utilizó un penetrómetro (Pesola Medio-Line Scales and presure set) para medir la fuerza ejercida necesaria para perforar la lámina foliar o la del folíolo en el caso de hojas compuestas. La medición se realizó en la zona media ubicada entre el borde de la lámina y la nervadura central, en condiciones de humedad de las hojas similares para todas las especies.

\section{Análisis de datos}

Para evaluar si existen diferencias entre grupos funcionales y entre especies en la composición y cantidad de mantillo se utilizaron modelos anidados, para evaluar los factores a distintos niveles. En estos modelos el factor ESPECIE se encuentra anidado en el factor GRUPO FUNCIONAL, de dos niveles (perennifolia y caducifolias, Zar, 1996). Las variables dependientes fueron la profundidad, el peso total del mantillo y de cada componente del mismo (hojarasca, ramas, hierbas y pastos, MOI $>1 \mathrm{~mm}$ y $\mathrm{MOI}<1 \mathrm{~mm}$, Facelli \& Pickett, 1991b), y la producción anual de material senescente (total y el peso de cada fracción del mismo). Para cada análisis se utilizó como covariable la altura y el área de dosel proyectada de cada arbusto para independizar el análisis del tamaño del arbusto. Ante el no cumplimiento de los supuestos se procedió a analizar transformando la variable o a través de análisis no paramétricos (Conover, 1980).

Para evaluar la heterogeneidad del mantillo debajo de las distintas especies se aplicó el índice de diversidad de Shannon-Wiener considerando los distintos componentes del mantillo y se comparó este índice entre grupos funcionales y entre las especies dentro de cada grupo a través de ANOVA 
anidado (Factor ESPECIE anidado en factor GRUPO FUNCIONAL, Zar, 1996). Además, se compararon las proporciones de las distintas fracciones del mantillo mediante un test exacto de Fisher (Quinn \& Keough, 2002).

Se compararon las variables morfológicas de la hojarasca: área foliar, peso foliar, dureza foliar y AFE entre grupos funcionales y entre las especies dentro de cada grupo mediante modelos lineales generalizados anidados (Factor ESPECIE anidado en Factor GRUPO FUNCIONAL), porque estas variables no se ajustaban a la distribución Normal. La distribución elegida para el análisis, Gamma, supone mayor variancia a mayores medias (Zar, 1996).

Se compararon las proporciones de hojarasca y ramas finas, tanto caídas anualmente como presentes en el mantillo, a través de test de $\chi^{2}$.

Tabla 2. Proporciones (\%) de las distintas fracciones del mantillo y del material senescente anual para los grupos funcionales y especies en estudio. Se indican las diferencias entre el mantillo y el material senescente anual en las proporciones de hojarascas y ramas finas.

\begin{tabular}{|c|c|c|c|c|c|c|c|c|c|c|}
\hline \multirow[b]{2}{*}{ Especie/Grupo } & \multicolumn{5}{|c|}{ Mantillo } & \multicolumn{3}{|c|}{ Material senescente/año } & \multicolumn{2}{|c|}{$\begin{array}{l}\text { Diferencia de } \\
\text { proporciones }\end{array}$} \\
\hline & Hojarasca & Ramas & MOl>1mm & MOl<1mm & Hierbas & Hojarasca & Ramas & Frutos & $x^{2}$ & $\mathbf{P}$ \\
\hline Caducifolias & 14,0 & 16,9 & 28,5 & 37,2 & 4,2 & 85,3 & 10,8 & 3,9 & 24,72 & $<0,01$ \\
\hline D. articulata & 2,3 & 26,8 & 22,5 & 46,0 & 2,4 & 62,2 & 30,1 & 7,7 & 35,06 & $<0,01$ \\
\hline D.chacaye & 11,3 & 5,4 & 32,9 & 49,3 & 1,1 & & & & & \\
\hline D.juncea & 0,4 & 49,0 & 18,6 & 30,5 & 1,5 & 68,7 & 20,6 & 10,7 & 73,90 & $<0,01$ \\
\hline E. virgata & 21,3 & 11,2 & 30,2 & 29,4 & 8,0 & & & & & \\
\hline N. antarctica & 20,8 & 5,3 & 32,3 & 36,4 & 5,1 & 99,2 & 0,8 & 0,0 & 8,80 & $<0,01$ \\
\hline R. rubiginosa & 28,1 & 3,8 & 34,6 & 31,5 & 2,0 & 99,6 & 0,4 & 0,0 & 5,74 & 0,02 \\
\hline R. cucullatum & 33,0 & 2,4 & 28,1 & 26,9 & 9,7 & 96,6 & 2,3 & 1,1 & 9,65 & 0,02 \\
\hline Perennifolias & 31,8 & 5,2 & 23,6 & 36,3 & 3,2 & 82,5 & 5,9 & 11,6 & 0,99 & 0,32 \\
\hline A. boronioides & 20,0 & 10,1 & 21,8 & 48,1 & 0,0 & & & & & \\
\hline A. integerrima & 19,1 & 5,0 & 30,5 & 45,3 & 0,2 & 95,9 & 4,0 & 0,1 & 8,15 & $<0,01$ \\
\hline B. microphylla & 22,6 & 5,4 & 28,2 & 39,2 & 4,6 & 84,5 & 0,1 & 15,4 & 12,15 & $<0,01$ \\
\hline B. obovata & 12,4 & 3,0 & 34,4 & 39,4 & 10,6 & & & & & \\
\hline E. coccineum & 37,5 & 2,8 & 26,6 & 30,3 & 2,8 & & & & & \\
\hline L. hirsuta & 80,6 & 0,8 & 7,5 & 9,5 & 1,5 & 75,0 & 1,1 & 23,9 & 0,01 & 0,96 \\
\hline M. boaria & 29,4 & 12,2 & 17,9 & 38,2 & 2,3 & 81,5 & 17,8 & 0,7 & 2,32 & 0,13 \\
\hline S. patagonicus & 32,8 & 2,0 & 21,6 & 40,3 & 3,4 & 75,7 & 6,4 & 17,9 & 0,08 & 0,78 \\
\hline
\end{tabular}

Tabla 3. Comparación del peso del mantillo acumulado y la producción de hojarasca material senescente anual de las especies del Matorral del NO de la Patagonia y en los micrositios de otros ambientes. Las letras indican las referencias.

\begin{tabular}{|lcc|}
\hline \multicolumn{1}{|c}{ Ambiente } & $\begin{array}{c}\text { Mantillo } \\
(\mathbf{g ~ m - 2 )}\end{array}$ & $\begin{array}{c}\text { Material senescente anual } \\
(\mathbf{g ~ m}-2 \text { año-1) }\end{array}$ \\
Matorral NO Patagonia & $348-2535^{\mathrm{a}}$ & $3-396^{\mathrm{a}}$ \\
Matorrales mediterráneos (Chile) & $7556^{\mathrm{b}}$ & $300-500^{\mathrm{k}}$ \\
Matorrales mediterráneos (España) & $1200^{\mathrm{c}}$ & $200-800^{\mathrm{l}}$ \\
Matorrales húmedos (China) & $1300-3700^{\mathrm{d}}$ & $231-409^{\mathrm{e}}$ \\
Chaparral & $182-263^{\mathrm{e}}$ & $140-400^{\mathrm{g}}$ \\
Monte Patagónico & $1600-8200^{\mathrm{f}, \mathrm{g}}$ & \\
Bosques mediterráneos & $200-1010^{\mathrm{h}}$ & $470-510^{\mathrm{m}}$ \\
Bosques de Nothofagus pumilio & & $280-400^{\mathrm{n}}$ \\
Bosque de Nothofagus spp. & $4300-5600^{\mathrm{i}}$ & $1600^{\circ}$ \\
\hline Bosques de Austrocedrus chilensis & $0-500^{\mathrm{j}}$ & \\
Bosque tropical & & \\
\hline
\end{tabular}

a Nuestros resultados, ${ }^{b}$ Cavieres et al. (2007), c Shengli et al. (2009), ${ }^{d}$ Kittredge (1939), e Carrera et al. (2009), ${ }^{\mathrm{f}}$ Pausas (1997), ${ }^{\mathrm{g}}$ Kavvadias et al. (2001), ${ }^{\mathrm{h}}$ Barrera et al. (2000)i Gobbi (2000), ' Dos Santos (2002), ${ }^{\mathrm{k}}$ Romanyà et al. (2000) y Fioretto et al. (2003), 'Gray (1982), ' Vivanco (2008), " Barrera et al. (2004), ${ }^{\circ}$ Bonilla et al. (2008). 


\section{M. del Paz et al. - Mantillo de especies leñosas de matorrales de la Patagonia}

Dichas comparaciones se realizaron para cada una de las especies y para los grupos funcionales de especies perennifolias y caducifolias.

En todas las pruebas estadísticas se estableció un $P<0,05$ como nivel de rechazo y las comparaciones múltiples se realizaron utilizando intervalos de confianza al $95 \%$.

\section{RESULTADOS}

\section{Profundidad y cantidad total de mantillo}

La profundidad del mantillo debajo de los individuos en estudio de todas las especies fue, en promedio de $2,7 \pm 0,3 \mathrm{~cm}$, con valores máximos de 8,4 $\pm 0,6 \mathrm{~cm}$ en $L$. hirsuta y mínimos bajo $D$. articulata $(<0,5 \mathrm{~cm})$, que forma una capa casi irreconocible de mantillo. Por esto, D. articulata no fue incluida en el análisis estadístico de la profundidad de mantillo. No se observó mantillo sobre el suelo fuera del dosel de las plantas leñosas en estudio.

El peso total del mantillo varió entre $347,9 \mathrm{gm}^{-2}$ y $2534,8 \mathrm{gm}^{-2}$ según las especies, y el área del dosel no influyó significativamente en la acumulación del mismo $\left(F_{1,125}=1,05 ; P=0,30\right.$, Fig. $\left.1 b\right)$. La cantidad de hojarasca de otras especies leñosas, hierbas y pastos captada fue prácticamente nula.

En términos generales, las especies perennifolias tuvieron mayor profundidad (Fig. 1a) y mayor peso total del mantillo (Fig. 1b) que las caducifolias (profundidades de $3,7 \pm 0,3$, y $2,1 \pm 0,3 \mathrm{~cm}, F_{1,126}=$ 23,$6 ; P<0,01$ respectivamente, y pesos de $1579,8 \pm$ 104,9 y $1066,6 \pm 100,2 \mathrm{gm}^{-2}, F_{1,134}=20,60 ; P<0,01$, respectivamente). Se encontraron diferencias entre especies del mismo grupo funcional tanto para la profundidad $\left(F_{12,126}=31,3 ; P<0,01\right)$ como para el peso total del mantillo $\left(F_{13,135}=7,2 ; P<0,01\right)$.

Los valores más altos en profundidad de mantillo (Fig. 1a) entre las perennifolias correspondieron a L. hirsuta y A. integerrima $(8,4 \pm 0,6$ y $5,8 \pm 0,4$ $\mathrm{cm}$, respectivamente) y el resto de las especies se diferenciaron gradualmente entre 1 y $2,9 \mathrm{~cm}$. Entre las especies caducifolias, $N$. antarctica y $R$. cucullatum tuvieron los valores más altos $(4,4$ $\pm 0,6$ y $3,3 \pm 0,5 \mathrm{~cm})$ y el resto de las especies se diferenció gradualmente entre 0 y $1,8 \mathrm{~cm}$ ).

Los valores mayores del peso total del mantillo (Fig. 1b) entre las especies perennifolias correspondieron a L. hirsuta y E. coccineum $(2377,9$ $\pm 222,7 \mathrm{gm}^{-2}$ y $2534,8 \pm 113,0 \mathrm{gm}^{-2}$, respectivamente) y los menores a $B$. microphylla y $B$. obovata (cercano a $500 \mathrm{gm}^{-2}$ ). Entre las especies caducas, $N$. antarctica tuvo los mayores valores $(1879,1 \pm$ 56,1; Fig. 1a) mientras que Discaria articulata, y $D$. juncea presentaron los menores valores de mantillo menores $\left(<800 \mathrm{gm}^{-2}\right)$.

Ninguna de las covariables (área de dosel, altura) probadas tuvo influencia sobre la profundidad y el total del mantillo $(P>0,05)$.

\section{Producción de material senescente por año}

La producción anual de material senescente de las especies estudiadas fue en promedio de 93,5 $\pm 1,9 \mathrm{gm}^{-2}$. Las especies perennifolias produjeron significativamente más material que las especies caducifolias $\left(143,2 \mathrm{gm}^{-2} \mathrm{año}^{-1}\right.$ y 44,8 $\mathrm{gm}^{-2} \mathrm{año}^{-1}$, respectivamente, $\left.F_{1,41}=23,3 ; P<0,01\right)$. En cada uno de los grupos funcionales se encontraron diferencias entre las especies (Fig. 1 c, $F_{9,41}=47,15 ; P<0,01$ ). Entre las especies perennifolias, A. integerrima, M. boaria, y L. hirsuta (entre 74 y $400 \mathrm{gm}^{-2} \mathrm{año}^{-1}$ ) fueron las especies con mayor aporte de material y B. microphylla y M. boaria las de menor aporte $\left(>16 \mathrm{gm}^{-2}\right)$. Entre las especies caducas, $N$. antarctica y $R$. cucullatum (entre 80 y $120 \mathrm{gm}^{-2} \mathrm{año}^{-1}$ ) tuvieron los valores más altos, mientras que $D$. articulata y D. juncea tuvieron los valores más bajos $\left(>8 \mathrm{gm}^{-}\right.$ $\left.{ }^{2} \mathrm{añon}^{-1}\right)$.

El peso de la hojarasca caída anualmente fue significativamente mayor en las especies perennifolias que en las caducifolias. $\left(F_{9,41}=36,1\right.$; $P<0,01)$. La hojarasca constituyó el componente más importante para la mayoría de las especies en estudio, excepto en $D$. articulata y $D$. juncea, especies con menor producción de material senescente anual, donde las ramas finas fueron el componente más representado (30 y 20,6 \% del total del mantillo/año, respectivamente), como así también los frutos (10\% y $14 \%$, respectivamente). Sin embargo, la cantidad de ramas finas y los frutos en el mantillo producidos en un año no mostraron diferencias significativas entre las especies perennifolias y caducifolias $\left(F_{9,41}=\right.$ 3,$13 ; P>0,08$ y $F_{6,44}=3,69 ; P=0,06$, respectivamente). De este último análisis se excluyeron $N$. antarctica y $M$. boaria, porque no se encontraron frutos en el material senescente anual. Entre las perennifolias, $L$. hirsuta, S. patagonicus y B. microphylla aportaron gran cantidad de frutos en el material senescente anual $(21,1 \%, 19,1 \%$ y $16,6 \%$ del total anual). 
Bol. Soc. Argent. Bot. 48 (3-4) 2013
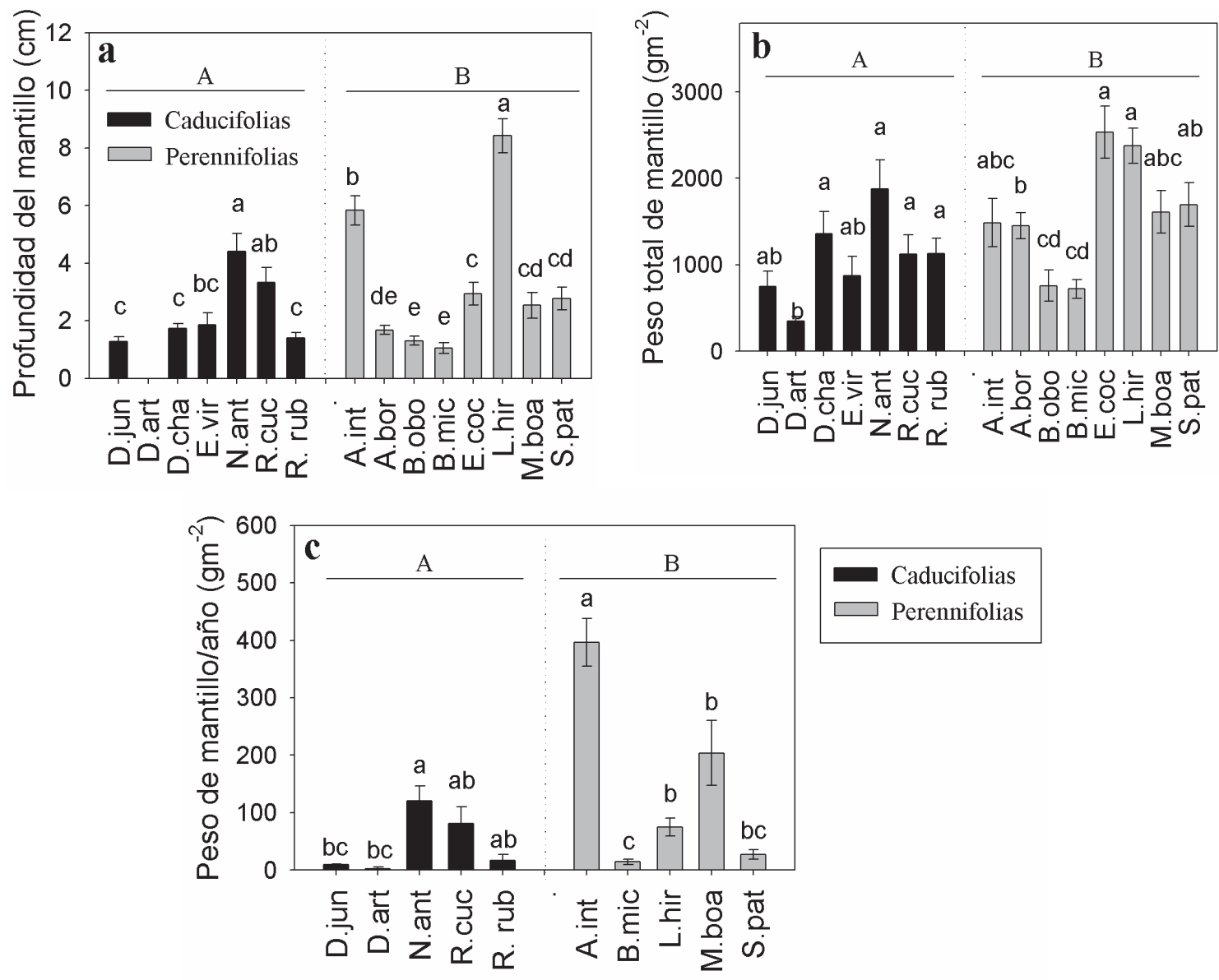

Fig. 1. Peso total (a) y profundidad del mantillo (b) y peso del material senescente/año (c), de las 15 especies en estudio: Caducifolias: D. jun=Diostea juncea, D.art=Discaria articulata, D.cha=D. chacaye, E. vir=Escallonia virgata, R.cuc=Ribes cucullatum, R.rub=Rosa rubiginosa; y Perennifolias: A.bor=Adesmia boronioides, A. int=Acaena integerrima, B.ovo=Baccharis obovata, B. mic=Berberis microphylla, E.coc= Embothrium coccineum, L.hir=Lomatia hirsuta, M.bor=Maytenus boaria, N.ant=Nothofagus antarctica, S.pat=Schinus patagonicus. Las barras de error corresponden al error estándar. Letras mayúsculas diferentes indican diferencias significativas entre grupos funcionales y letras minúsculas diferentes indican diferencias entre especies dentro de cada grupo funcional $(P<0,05)$.

\section{Composición del mantillo}

El peso estimado total de la hojarasca fue 413,7 $\pm 69,9 \mathrm{gm}^{-2}$, y representa el $26,8 \%$ del mantillo. Las especies perennifolias tuvieron, en promedio, mayores pesos de hojarasca en el mantillo que las especies caducifolias $\left(592,1 \pm 71.1 \mathrm{gm}^{-2}\right.$ y $214,6 \pm$ $36,4 \mathrm{gm}^{-2}$, respectivamente, $F_{1,135}=67,2 ; P<0.01$ ). Asimismo, las especies perennifolias mostraron menor variación que las caducifolias en el peso de hojarasca ( $\mathrm{CV}=107,5$ y 144,1, respectivamente) y se encontraron diferencias entre las especies de cada uno de los grupos funcionales $\left(F_{13,135}=26,09\right.$; $P<0,01)$. Entre las especies perennifolias, $L$. hirsuta y E. coccineum presentaron la mayor cantidad de hojarasca (entre 950 y $1920 \mathrm{gm}^{-2}$, Fig. 2a), y el resto se diferenciaron gradualmente entre los 94, 6 y 552,6 $\mathrm{gm}^{-2}$. Entre las especies caducifolias, hay una diferenciación gradual en el peso de la hojarasca entre $R$. cucullatum y $N$. antarctica donde se hallaron los valores más altos $\left(378,0 \pm 160,5 \mathrm{gm}^{-2}\right.$ y 527,0 $\pm 107,2$, respectivamente $\left.\mathrm{gm}^{-2}\right)$ y $D$. juncea y $D$. articulata donde fue casi imperceptible $\left(<2,8 \mathrm{gm}^{-2}\right)$. 


\section{M. del Paz et al. - Mantillo de especies leñosas de matorrales de la Patagonia}

Las ramas finas presentes en el mantillo pesaron, en promedio, 92,7 $\pm 14,3 \mathrm{~g} \mathrm{~m}^{-2}$, que representa aproximadamente un $10 \%$ del mantillo total de las especies en estudio. Las especies perennifolias $\left(80,0 \pm 1,3 \mathrm{gm}^{-2}\right)$ y las caducifolias $(121,9 \pm 3,2$ $\left.\mathrm{gm}^{-2}\right)$ tuvieron similar cantidad promedio de ramas $\left(F_{1,135}=2,5 ; P=0,11\right)$. Se observó similar variabilidad en ambos grupos (CV Perennifolias $=131,0 \%$; CV Caducifolias $=188,0 \%$ ). Diostea juncea y M. boaria $(352,1 \pm 152,1$ y $209,1 \pm 55,8$, respectivamente $\mathrm{gm}^{-2}$ ) fueron las especies con mayor peso de ramas, mientras que L. hirsuta, $R$. cucullatum, $S$. patagonicus, $B$. ovobata y $B$. microphylla tuvieron los valores medios más bajos (entre 23,8 y $40,4 \mathrm{gm}^{-}$ $\left.{ }^{2}\right)$. Discaria articulata tuvo casi un tercio del total de su mantillo constituido por ramas finas (Fig. 2b).

La MOI $>1 \mathrm{~mm}$ constituyó en promedio el 25 $\%$ del mantillo $\left(329 \pm 74 \mathrm{gm}^{-2}\right)$ de las especies en estudio (Fig. 2c). Las especies perennifolias tuvieron similar MOI $>1 \mathrm{~mm}$ que las caducifolias $(342.5 \pm 4,4$ y $316,8 \pm 3,2 \mathrm{gm}^{-2}$, respectivamente, $F_{1,135}=0,42$; $P=0,52)$. Nothofagus antarctica y $E$. coccineum tuvieron los valores más altos de MOI $>1 \mathrm{~mm}(>675$ $\mathrm{gm}^{-2}$, respectivamente), mientras que $D$. articulata, $D$. juncea y $L$. hirsuta tuvieron los valores más bajos (entre 69 y $186 \mathrm{gm}^{-2}$ ).

El peso de la MOI más particulada $(\mathrm{MOI}<1 \mathrm{~mm})$ constituyó en promedio un $36 \%$ del total del mantillo (aproximadamente 455,8 $\pm 33,0 \mathrm{gm}^{-2}$, Fig. 2d). Las especies perennifolias tuvieron mayor $\mathrm{MOI}<1 \mathrm{~mm}$ que las caducifolias $\left(529 \pm 126\right.$ y $311 \pm 117 \mathrm{gm}^{-2}$, respectivamente, $\left.F_{1,135}=7.2 ; P<0.01\right)$. Asimismo, se encontraron diferencias entre las especies de cada uno de los grupos funcionales $\left(F_{13,135}=2,96 ; P<0,01\right)$. Entre las especies perennifolias $E$. coccineum y $S$. patagonicus tuvieron los valores más altos de $\mathrm{MOI}<$ $1 \mathrm{~mm}$ y $L$. hirsuta, B. microphylla y B. ovobata los valores más bajos. En las especies caducifolias existió una diferenciación gradual entre 137 y 615,3 gm $^{-2}$ (Fig. 2d).

La cantidad de hierbas y pastos presente en el mantillo fue similar entre perennifolias $\left(39,9 \pm 0,9 \mathrm{gm}^{-}\right.$ $\left.{ }^{2}\right)$ y caducifolias $\left(44,5 \pm 0,9 \mathrm{gm}^{-2}, F_{1,135}=0,20 ; P=0,65\right)$, formándose un gradiente entre $R$. cucullatum, $E$. coccineum, y E. virgata con valores altos de hierbas y pastos (entre 70 y $110 \mathrm{gm}^{-2}$ ) y $A$. boronioides, $A$. integerrima y $D$. articulata y $D$. juncea con valores muy bajos (valores $<11 \mathrm{gm}^{-2}$, Fig. 2e).

La heterogeneidad del mantillo fue similar entre especies perennifolias y caducifolias $\left(F_{1,135}=3,2\right.$;
$P=0,07$, Fig. 3). La mayoría de las especies tuvo valores altos de heterogeneidad de mantillo y similares entre sí $(1,07 \pm 0,5), D$. juncea, tuvo valores intermedios $(0,86 \pm 0,07)$ y Lomatia hirsuta tuvo la menor heterogeneidad del mantillo $(0,64 \pm 0,75)$. La abundancia de los componentes del mantillo fue diferente según la especie (Tabla 2). Por ejemplo, L. hirsuta mostró los valores más altos de hojarasca $(>$ al $80 \%$ del total del mantillo), aproximadamente el $50 \%$ del total del mantillo de $D$. juncea está formado por pequeñas ramas finas, y la MOI $<1 \mathrm{~mm}$ en $D$. articulata, $D$. chacaye y A. boronioides representó más del $45 \%$ de la hojarasca. El resto de las especies tuvieron porcentajes similares de los distintos componentes del mantillo. Las especies caducifolias poseen más del doble de ramas finas y $60 \%$ más de hierbas $\mathrm{y}$ pastos en su mantillo que las perennifolias $\left(F_{8,7}=0,16 ; P<0,01\right.$ y $\left.F_{8,7}=-0,19 ; P=0,61\right)$, mientras que estas últimas tienen el doble de hojas en su mantillo que las especies caducifolias $\left(F_{8,7}=0,19\right.$; $P<0,01$, Tabla 2).

Ninguna de las covariables probadas (altura, área de dosel y dureza) tuvo influencia en peso de las distintas fracciones del mantillo $(P>0,05)$.

\section{Características foliares de la hojarasca}

El área foliar de las especies de matorral es en promedio de $287,6 \pm 25,5 \mathrm{~mm}^{2}$ (CV 173,9). Las especies perennifolias tuvieron en promedio mayor área foliar que las caducifolias $(455,0 \pm 7,9$ y $\left.95,5 \pm 0,9 \mathrm{~mm}^{2}, F_{1,135}=160,3 ; P<0,01\right)$ y con más variabilidad (CV=139,9 y 70,5, respectivamente). Asimismo, se encontraron diferencias entre las especies de cada uno de los grupos funcionales $\left(F_{13,135}=18,1 ; P<0,01\right)$. Entre las perennifolias, $L$. hirsuta tuvo las hojas más grandes $(2010,7 \pm 153,1$ $\left.\mathrm{mm}^{2}\right)$, B. obovata y B. microphylla tuvieron las hojas más chicas $\left(72,2 \pm 3\right.$ y $74,4 \mathrm{~mm}^{2}$, respectivamente) y el resto de las especies perennifolias se diferenció gradualmente entre 138,0 y $539,4 \mathrm{~mm}^{2}$. Entre las especies caducifolias, la mayoría de las especies se diferenciaron entre 62,0 y $185,7 \mathrm{~mm}^{-2}$, excepto $D$. articulata y E. virgata que tuvieron las hojas más chicas $\left(<38,7 \mathrm{~mm}^{2}\right.$, respectivamente, Fig. $\left.4 \mathrm{a}\right)$.

El peso foliar promedio fue $0,07 \pm 0,01 \mathrm{~g}$, muy variable entre especies (CV=192,7; Fig. 4b) y mayor en especies perennifolias que en caducifolias $(0,10$ y $0,03 \mathrm{~g}$, respectivamente, $\left.F_{1.148}=43,2 ; P<0,01\right)$. Asimismo, se encontraron diferencias entre las 
Bol. Soc. Argent. Bot. 48 (3-4) 2013
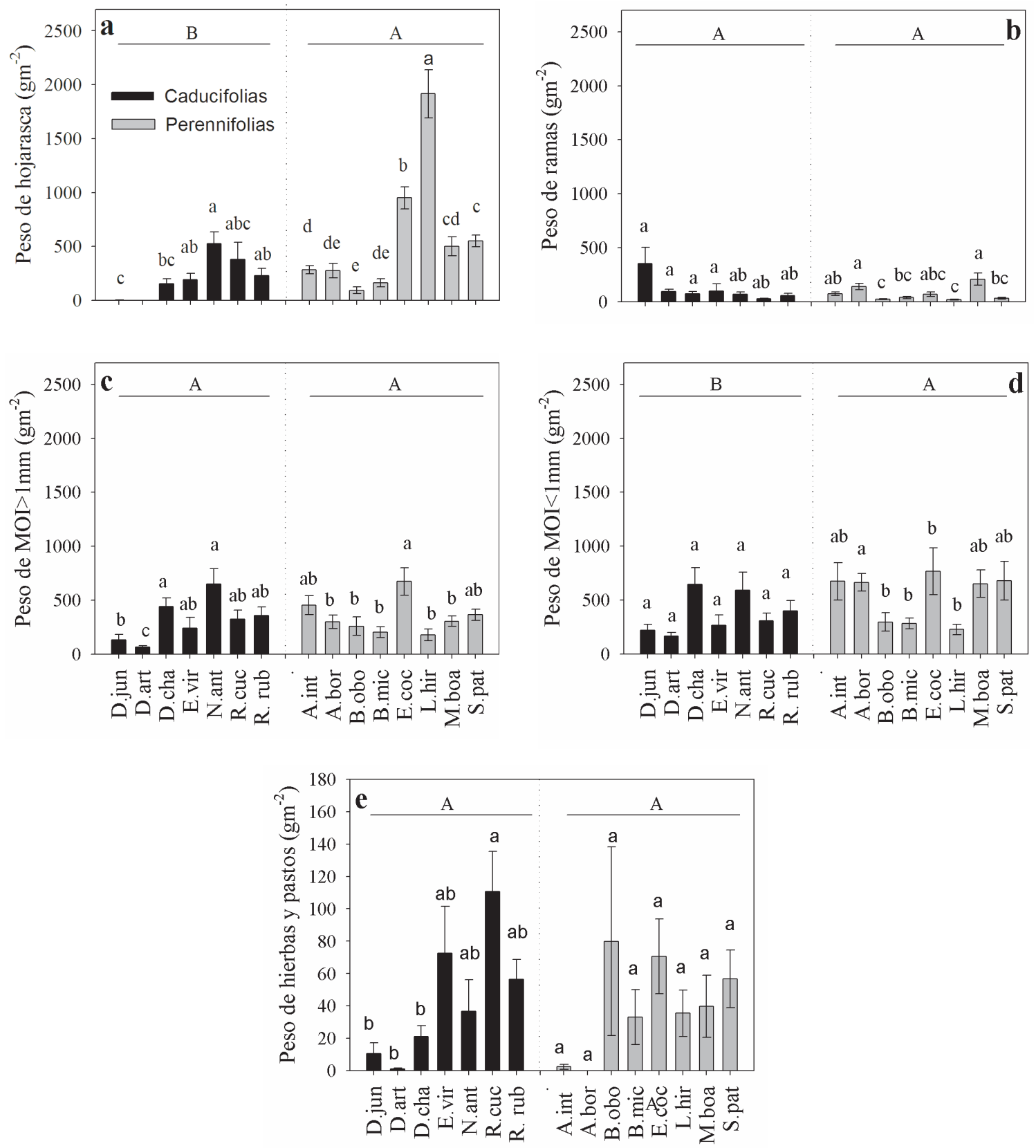

Fig. 2. Composición del mantillo, presentado a través del peso de hojarasca (a), ramas finas $(<1 \mathrm{~cm}$ de diámetro) (b), Materia orgánica irreconocible (MOI) mayor a $1 \mathrm{~mm}=\mathrm{MOI}>1 \mathrm{~mm}$ (c) y menor a 1 $\mathrm{mm}=\mathrm{MOI}<1 \mathrm{~mm}(\mathbf{d})$ hierbas y pastos $(\mathbf{e})$, de las 15 especies estudiadas: Caducifolias: $D$. jun=Diostea juncea, D. art=Discaria articulata, D.cha $=D$. chacaye, E. vir=Escallonia virgata, R.cuc=Ribes cucullatum, R.rub=Rosa rubiginosa; $y$ Perennifolias: A.bor=Adesmia boronioides, $A$. int=Acaena integerrima, B.ovo= Baccharis obovata, B. mic=Berberis microphylla, E.coc=Embothrium coccineum, L. hir=Lomatia hirsuta, M.bor=Maytenus boaria, N.ant=Nothofagus antarctica, S.pat=Schinus patagonicus. Las barras de error corresponden al error estándar. Letras mayúsculas diferentes indican diferencias significativas entre grupos de especies y letras minúsculas indican diferencias entre especies dentro de cada grupo $(P<0,05)$. 
especies de cada uno de los grupos funcionales $\left(F_{13,135}=35,9 ; P<0,01\right)$. Entre especies perennifolias, las hojas más pesadas fueron las de $L$. hirsuta $(0,41$ $\pm 0,04 \mathrm{~g})$, las más livianas las de $B$. microphylla $(<0,017 \mathrm{~g})$ y el resto de las especies se diferenció gradualmente entre 0,029 y $0,070 \mathrm{~g}$. En tanto que entre las especies caducifolias, se conformó un gradiente entre $R$. rubiginosa $(0,15 \pm 0,04 \mathrm{~g})$, que tuvo las hojas más pesadas, y $D$. articulata y $E$. virgata las hojas más livianas (<0,004 g, Fig. 4b).

La dureza foliar promedio fue de 137,6 $\pm 4,9$ $\mathrm{gmm}^{-2}$ y mayor en especies perennifolias que en caducifolias $\left(136,6 \pm 0,9\right.$ y $105,8 \pm 0,7 \mathrm{gmm}^{-2}$, respectivamente, $\left.F_{1,135}=138,1 ; P<0,01\right)$. Asimismo, se encontraron diferencias entre las especies de cada uno de los grupos funcionales $\left(F_{11,117}=75,1\right.$ : $P<0,01)$. Entre las perennifolias la mayor dureza foliar se registró en L. hirsuta $\left(270,0 \pm 9,3 \mathrm{gmm}^{-}\right.$ $\left.{ }^{2}\right)$ y la menor en $E$. coccineum y A. integerrima $\left(<95\right.$ gmm $^{-2}$, Fig.4c). Entre las caducifolias, y $R$. rubiginosa y $N$. antarctica presentaron las hojas más duras $\left(171,1 \pm 6,3\right.$ y $160,9 \pm 4,5 \mathrm{gmm}^{-2}$, respectivamente Fig. $4 \mathrm{c})$, y $D$. chacaye, $D$. juncea, y $R$. cucullatum, las hojas más blandas $(<84,0$ $\mathrm{gmm}^{-2}$; respectivamente). Por limitaciones del método de medición no se midió la dureza de las hojas de D. articulata y A. boronioides.

El AFE promedio de las especies estudiadas fue $64,9 \pm 3,0 \mathrm{~mm}^{2} \mathrm{~g}^{-1}$ siendo las especies significativamente menor en las especies perennifolias respecto de las caducifolias $(53,3 \pm 0,3$ y $78,1 \pm 0,6 \mathrm{~mm}^{2} \mathrm{~g}^{-1}$, respectivamente, $F_{1,134}=25,3$; $P<0,01)$. Asimismo, se encontraron diferencias entre las especies de cada uno de los grupos funcionales $\left(F_{13,135}=5,2 ; P<0,01\right)$. Entre las especies perennifolias, existe una diferenciación gradual entre especies desde $M$. boaria con los valores más altos $\left(88,1 \pm 7,1 \mathrm{~mm}^{2} \mathrm{~g}^{-1}\right)$, y A. boronioides, L. hirsuta, y $A$. integerrima con valores más bajos $\left(<42 \mathrm{~mm}^{2} \mathrm{~g}^{-1}\right.$, respectivamente, Fig. $\left.4 \mathrm{~d}\right)$. También observó una diferenciación gradual en las especies caducifolias, desde $N$. antarctica y E.virgata con valores más altos (111,3 y $108 \mathrm{~mm}^{2} \mathrm{~g}-$ ${ }^{1}$, respectivamente) hasta $D$. articulata con valores más bajos $\left(46,0 \pm 6,9 \mathrm{~mm}^{2} \mathrm{~g}^{-1}\right.$, Fig. $\left.4 \mathrm{~d}\right)$.

Relación entre material senescente anual y el mantillo

En las especies caducifolias, el material senescente anual posee $70 \%$ de hojarasca y un $10 \%$ de ramas (Tabla
2). Estás proporciones difieren significativamente de lo encontrado en el mantillo (16\% hojarasca y $17 \%$ ramas, $\left.\chi^{2}=19,6 ; P<0,01\right)$. En tanto que en las especies perennifolias el material senescente anual y el mantillo no difieren en las proporciones de hojarasca (82,5 y $37,1 \%$, respectivamente) y ramas (6 y $5 \%$, respectivamente). A nivel de especies se distinguen tres patrones (Tabla 2), a) en $S$. patagonicus, $M$. boaria, L. hirsuta no existen diferencias en las proporciones de hojarasca y ramas entre el material senescente anual y el mantillo, b) D. juncea es significativamente mayor la proporción de ramas y menor la proporción de hojarasca en el mantillo que en el material senescente anual $\left(\chi^{2}=73,9 ; P<0,01\right)$ y c) en el resto de las especies la proporción de hojarasca

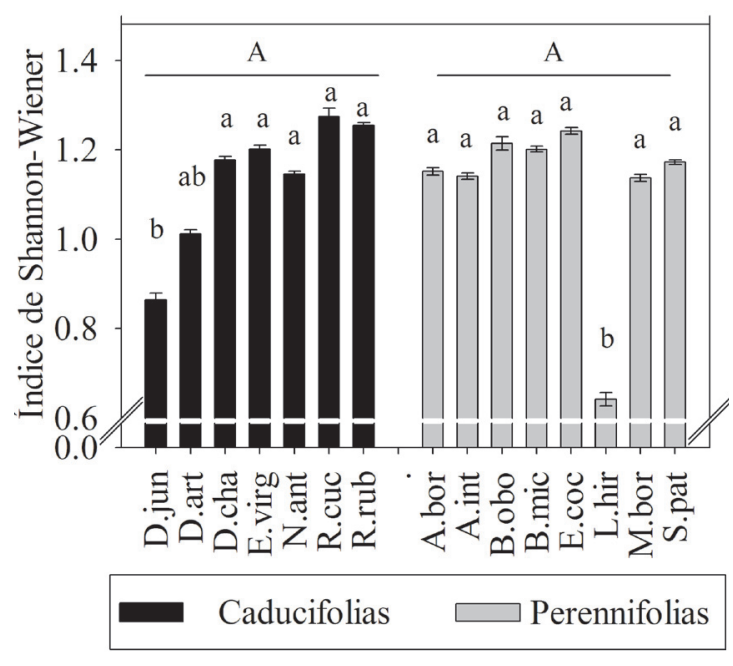

Fig. 3. Heterogeneidad del mantillo medido por el Índice de Shannon y Wiener aplicado a las distintas porciones del mantillo (hojarasca, ramas finas, hierbas y pastos, $\mathrm{MOI}<1 \mathrm{~mm}$ y $\mathrm{MOI}>1 \mathrm{~mm}$ ) de las 15 especies de estudio: Caducifolias: D. jun=Diostea juncea, D.art=Discaria articulata, D.cha $=D$. chacaye, E. vir=Escallonia virgata, R.cuc=Ribes cucullatum, R.rub=Rosa rubiginosa; y Perennifolias: A.bor=Adesmia boronioides, A. int=Acaena integerrima, B.ovo=Baccharis obovata, B. mic=Berberis microphylla, E.coc=Embothrium coccineum, L.hir=Lomatia hirsuta, M.bor= Maytenus boaria, N.ant=Nothofagus antarctica, S.pat=Schinus patagonicus . Las barras de error corresponden al error estándar. Letras mayúsculas diferentes indican diferencias significativas entre grupos de especies y letras indican minúsculas diferencias entre especies dentro de cada grupo $(P<0,05)$. 
Bol. Soc. Argent. Bot. 48 (3-4) 2013
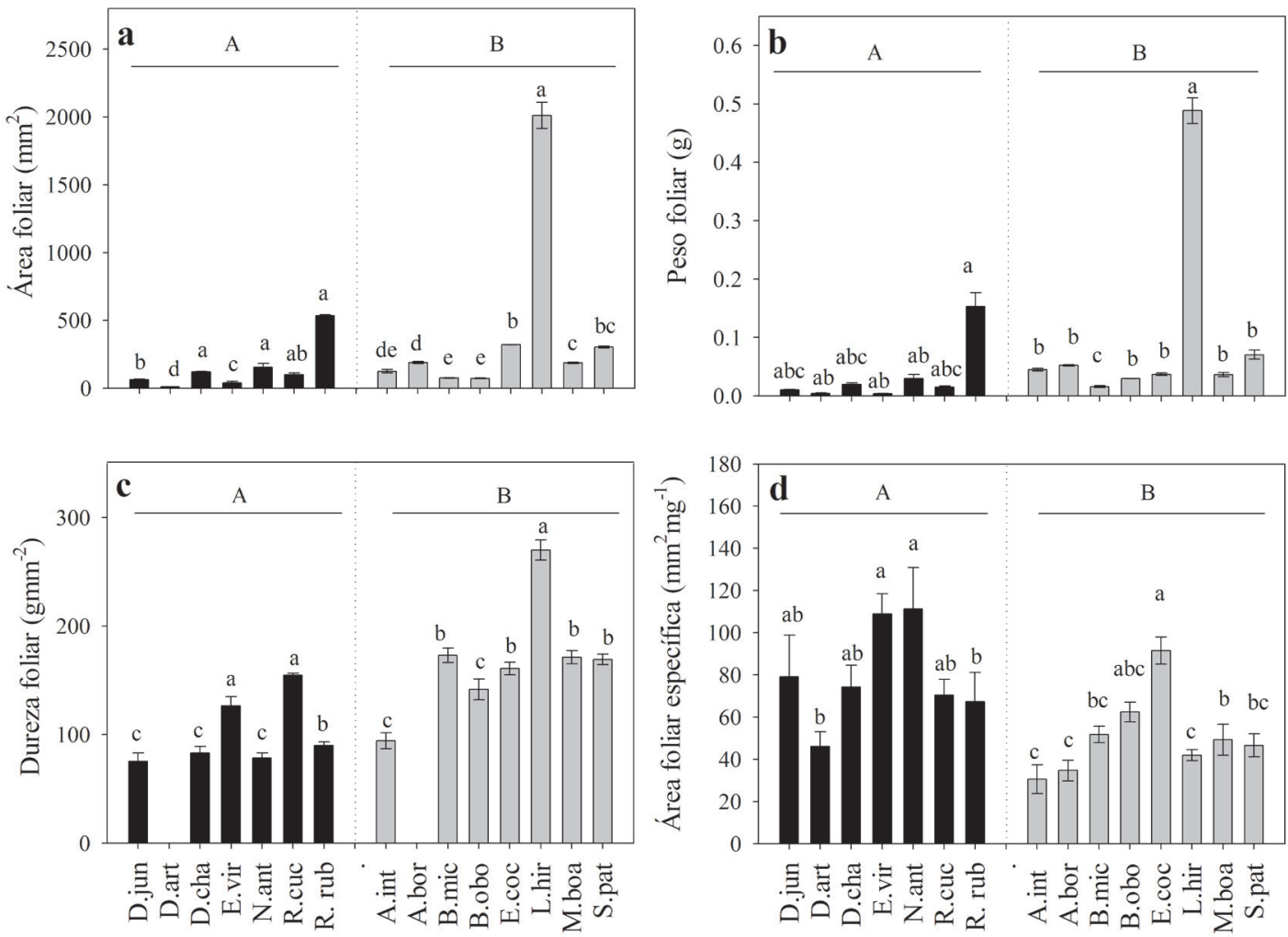

Caducifolias

$\square$ Perennifolias

Fig. 4. Propiedades de las hojas senescentes: área foliar (a), peso foliar (b), dureza foliar (c), área foliar específica (d) de las especies en estudio: Caducifolias: D. jun=Diostea juncea, D.art=Discaria articulata, D.cha=Discaria. chacaye, E. vir=Escallonia virgata, R.cuc=Ribes cucullatum, R.rub=Rosa rubiginosa. Perennifolias A.bor=Adesmia boronioides, A. int=Acaena integerrima, B.ovo=Baccharis obovata, B. mic= Berberis microphylla, E.coc=Embothrium coccineum, L.hir=Lomatia hirsuta, M.bor=Maytenus boaria, N.ant= Nothofagus antarctica, S.pat=Schinus patagonicus. Las barras de error corresponden al error estándar. Letras mayúsculas diferentes indican diferencias significativas entre grupos de especies y letras minúsculas diferentes indican diferencias dentro de cada grupo $(P<0,05)$.

en el mantillo fue significativamente menor que en el material senescente anual $\left(\chi^{2}=[D\right.$. Articulata $=35,1 ; N$. antarctica $=8,8 ; R$. rubiginosa $=5,7 ; R$. cucullatum $=$ $9,6 ;$ A. integerrima $=8,1 ; B$. microphylla $=12,1]$; $P<0,01)$.

\section{Discusión y Conclusiones}

El mantillo total acumulado por las especies más frecuentes de los matorrales del NO de la Patagonia varió entre 347,9 y $2534,7 \mathrm{gm}^{-2}$ (correspondiendo a D. articulata y E. coccineum respectivamente). Los valores registrados para distintos tipos de matorrales son muy variables y los obtenidos en este estudios están dentro del rango de los mencionados y son relativamente bajos comparados con bosques de esta región (Tabla 3). La producción media de material senescente por año fue baja $\left(93,5 \mathrm{gm}^{-2}\right)$ en comparación con otros ecosistemas, en particular debido a la presencia de especies (D. juncea y D. articulata) con valores mínimos $\left(<9 \mathrm{gm}^{-2}\right)$. Estos resultados indican una relación entre producción anual de material senescente y mantillo acumulado menor que la que se estima para los otros sistemas considerados y podría ser atribuida a bajas temperaturas en invierno 


\section{M. del Paz et al. - Mantillo de especies leñosas de matorrales de la Patagonia}

y estación seca en verano que podrían producir bajas tasas de descomposición (Tabla 3 ) y a menores tasas de fotodegradación que en ambientes con mayor radiación como la zona del monte patagónico (Austin \& Vivanco, 2006).

En el aporte anual de material senescente, $A$. integerrima, a pesar de ser el arbusto de menor tamaño relevado, constituyó una de las mayores contribuciones que son dos a tres veces superiores a especies arbóreas o arbustivas de gran tamaño. Esta especie tiene la particularidad de iniciar la descomposición cuanto la hojarasca aún está adherida a la planta; observaciones de campo muestran que la materia muerta representa el doble del peso que la parte aérea viva.

Las especies perennifolias aportan un 55,7\% del total del mantillo acumulado y un $61,5 \%$ del total de material senescente anual (promedios ponderados por la abundancia relativa de las especies en el lugar de estudio), a pesar de que representan un $42,8 \%$ de la cobertura versus un $48,2 \%$ en las especies caducifolias. Esto se atribuye principalmente a que entre las perennifolias se encuentran E. coccineum, $L$. hirsuta y $S$. patagonicus, tres de las cuatro especies con mayor mantillo acumulado, y A. integerrima y $M$. boaria que realizaron los mayores aportes de material senescente anual.

La proporción de hojarasca en el material senescente producido anualmente está dentro de los rangos mencionados para matorrales mediterráneos (67 y 85\%) (Romanyà et al., 2000; Fioretto et al., 2003) y los valores más bajos encontrados, que corresponden a $D$. articulata y D. juncea, $(62$ a $68,7 \%)$ son similares a los mencionados para los bosques de Nothofagus de la región (Vivanco, 2008). En general las especies perennifolias tienden a presentar una relación hojarasca caída anualmente/hojarasca en el mantillo (Ha/Hm) menor que las caducifolias (Olson, 1963), esto se atribuye a que suelen poseer hojas más pobres en nutrientes y/o con formas de carbono más recalcitrantes, menor biomasa microbiana en sus suelos y descomposición más lenta (Vitousek, 1982; Adams \& Attiwil, 1986; Vitousek \& Sanford, 1986; Aerts, 1996; Diehl et al., 2003; Vivanco, 2008). Los resultados obtenidos en este trabajo $(\mathrm{Ha} /$ $\mathrm{Hm}=0,36$ y 10,00 para perennifolias y caducifolias, respectivamente) parecen coincidir con esta tendencia. Entre las especies caducifolias, $D$. juncea y $D$. articulata tienen los mayores valores $\mathrm{Ha} / \mathrm{Hm}(46,5$ y 2,9 , respectivamente), y se diferencian claramente del resto de las especies por un bajísimo aporte de hojarasca anual y prácticamente nula acumulación de hojarasca en el mantillo. Sin embargo, las especies perennifolias y caducifolias no se diferencian en la acumulación del material proveniente de la primera fragmentación del mantillo (MOI $>1 \mathrm{~mm}$.) indicando que en las perennifolias hay un retraso en la fragmentación inicial del material senescente y esto se asocia a que poseen mayor dureza y menor AFE. Estas características foliares se asocian a mayores contenidos de lignina, celulosa y hemicelulosa en la hojas senescentes y a mayor longevidad de las mismas (Wright et al., 2004), por lo tanto han sido utilizadas como indicadoras de bajas tasas de descomposición de hojarasca (Lusk et al., 2001; Lambers et al., 2008). Por ello, encontramos similares proporciones de hojarasca en el material senescente anual que en el mantillo, en tres especies perennifolias (L. hirsuta, $M$. boaria y $S$. patagonicus) y diferentes proporciones en todas las especies caducas. Esto indica que las especies perennifolias invierten más en la producción de defensas estructurales (e.g., dureza de las hojas) mientras que las especies caducifolias en desarrollar su superficie fotosintética. Asimismo, las especies perennifolias acumulan más $\mathrm{MOI}<1 \mathrm{~mm}$, porque hay mayor cantidad de material senescente aportado y la descomposición de los compuestos orgánicos que le dan dureza a las hojas es más recalcitrante y de descomposición más lenta.

En casi todas las variables estudiadas se encontró una alta variabilidad entre las especies consideradas dentro de cada grupo funcional (diferencias graduales entre especies). Esto hace que haya especies perennifolias y caducifolias con mantillos y hojarasca de características similares. Podría plantearse que el grupo funcional conformado por especies caducifolias se subdivide en uno constituido por especies de menor abundancia de mantillo, mayor proporción de ramas finas, menor dureza, área y peso foliar $(D$. articulata y D. juncea) y otro formado por el resto de las especies caducas con mayor acumulación de mantillo, dureza foliar y AFE intermedias $(D$. chacaye, E. virgata, N. antarctica, $R$. cucullatum y $R$. rubiginosa). En el grupo funcional de las especies perennifolias se constituyen por un subgrupo similar al anterior (mayor acumulación de mantillo y AFE intermedias) salvo en la permanencia del follaje y la dureza de las hojas (A. integerrima, A. boronioides, $B$. ovobata, B. microphylla, M. boaria, S. patagonicus) y otro constituido exclusivamente por $L$. hirsuta, que 
posee la hojarasca más recalcitrante y el mantillo más homogéneo.

$\mathrm{La}$ ausencia de material originado a partir de hierbas y pastos en las trampas y la similitud en la acumulación de este material en el mantillo de ambos grupo funcionales indicaría que el mismo proviene de las plantas que crecen bajo el dosel de estos arbustos. Entonces las diferencias entre especies del mismo grupo funcional se relacionarían con las diferencias en la calidad como nodrizas de estas especies (Gutiérrez y Squeo, 2004).

Los incendios constituyen un disturbio frecuente e importante en el NO de Patagonia y su relación con los matorrales es muy estrecha (Veblen et al., 2011). Las características morfológicas de muchas especies les dan ventajas frente a determinados regímenes de fuego (Don Bradshaw et al. 2011; Keeley et al. 2011), muchas de esas características se asocian al material senescente y al mantillo. En un estudio reciente (Blackhall, 2012) realizado en seis especies arbustivas también consideradas en este trabajo, encontró modificaciones en las características foliares de especie que rebrotan post-fuego. En este sentido, comparando entre plantas rebrotantes post-fuego versus aquellas instaladas en sitios con incendios antiguos, todas las especies estudiadas tuvieron menor AFE, humedad y área foliar, la mayoría de las especies tuvo mayor tiempo de ignición y en particular las caducifolias tuvieron mayores peso y dureza foliar. De manera que, la similitud entre algunas características del grupo conformado por $R$. cucullatum, $N$. antárctica y $R$. rubiginosa con la mayoría de las especies perennifolias podría ser efecto del fuego reciente en nuestra área de estudio (10 años). Se ha encontrado que las hojas senescentes más grandes conforman una hojarasca más inflamable ya que producen un mantillo menos compacto y con mas oxígeno (Cornelissen et al., 2003; Scarff \& Westoby, 2006). En este sentido, las especies perennifolias al aportar más hojarasca anualmente, más persistente y de mayor área (>mantillo) podrían incrementar la probabilidad e intensidad del fuego.

En conclusión, las especies perennifolias y caducifolias se diferenciaron en la cantidad, composición y estructura del material senescente anual y del mantillo aunque no difirieron en la heterogeneidad de este último. En cada uno de los grupos funcionales considerados se encontraron especies con características particulares y contrastantes, $\mathrm{y}$ otras que representan un gradiente de variación.
Esto contribuye a proponer que en este tipo de matorrales existe una amplia diversidad de micrositios asociados a la hojarasca, que generan un gradiente de situaciones microambientales, que otorgan un abanico de oportunidades de establecimiento y hábitats a una gran diversidad de organismos. Esta diversidad de micrositios podrían tener implicancias en la dinámica de nutrientes (micrositios con alta y con bajas tasas de descomposición), en la protección del suelo contra la erosión (micrositios con alta cobertura y por lo tanto protegidos contra la erosión hídrica y eólica vs micrositios expuestos) y en la respuesta a los disturbios. Es necesario profundizar el efecto de las distintas porciones del mantillo en la descomposición, facilitación para la regeneración y en la acumulación de combustible.

\section{Agradecimientos}

Agradecemos a Carruitero M. y Aput, L. por su colaboración en los muestreos. Esta investigación se realizó en el marco del proyecto "Patrones y Procesos Ecológicos del Bosque Templado de Sudamérica Austral: variación geográfica, efectos sinérgicos disturbio-invasión y pulsos endógenos" financiado por la Universidad del Comahue (B0126).

\section{Bibliografía}

ADAMS, M.A. \& P.M. ATTIWIL. 1986. Nutrient cycling and nitrogen mineralization in eucalypt forests of south-eastern Australia. II: Indices of nitrogen mineralization. Plant Soil 92: 341-362.

AERTS, R. 1996. Nutrient resorption from senescing leaves of perennials: Are there general patterns? $J$. Ecol. 84: 597-608.

AERTS, R. 1997. Climate, leaf litter chemistry and leaf litter decomposition in terrestrial ecosystems: a triangular relationship. Oikos 79: 439-449

AGUIAR, M.R., A. SORIANO \& O.E. SALA. 1992. Competition and facilitation in the recruitment of seedlings in Patagonian steppe. Funct. Ecol. 6: 66-70.

ALAUZIS, M.V., M.J. MAZZARINO, E. RAFFAELE \& L. ROSELLI. 2004. Wildfires in NW Patagonia: long-term effects on a Nothofagus forest soil. Forest Ecol. Manag. 192: 131-142.

AUSTIN, A.T. \& L. VIVANCO. 2006. Plant litter decomposition in a semi-arid ecosystem controlled by photodegradation. Nature 442: 555-558. 


\section{M. del Paz et al. - Mantillo de especies leñosas de matorrales de la Patagonia}

BARRERA, M., J.L. FRANGI, J.J. FERRANDO \& J.F. GOYA. 2004. Descomposición del mantillo y liberación foliar neta de nutrientes de Austrocedrus chilensis (D. Don) Pic. Serm. et Bizzarri en El Bolsón, Río Negro. Ecol. Austral. 14: 99-112.

BASKIN, J.M. \& C.C. BASKIN. 1989. Physiology of dormancy and germination in relation to seed bank ecology. In: LECK, M.A., PARKER, V.T. \& R.L. SIMPSON (eds.), Ecology of Soil Seed Banks, pp. 54-65. Academic Press, London.

BERENDSE, F., B. BERG \& E. BOSATTA. 1987. The effect of lignin and nitrogen on the decomposition of litter in nutrient-poor ecosystems: a theoretical approach. Can. J. Bot. 65: 1116-1120.

BLACKHALL, M. 2012. Respuestas de especies leñosas a herbívoros e incendios en bosques y matorrales del noroeste de la Patagonia: Estudio de la inflamabilidad vegetal. p. 184. Universidad Nacional del Comahue. Centro Regional Universitario Bariloche, S. C. de Bariloche.

BONILLA, R., B. RONCALLO, J. JIMENO \& T. GARCÍA. 2008. Producción y descomposición de la hojarasca en bosques nativos y de Leucaena sp., en Codazzi, Cesar. Revista Corpoica - Ciencia y Tecnología Agropecuaria 9: 5-11.

CARMONA, M.R., M. AGUILERA, C.A. PEREZ \& I. SEREY. 2006. Actividad respiratoria en el horizonte orgánico de suelos de ecosistemas forestales del centro y sur de chile. Gayana Bot. 63: 1-12.

CARRERA,A.L., M.J. MAZZARINO, M.B. BERTILLER, H.F. DEL VALLE \& E.M. CARRETERO. 2009. Plant impacts on nitrogen and carbon cycling in the Monte Phytogeographical Province, Argentina. J. Arid Environ. 73: 192-201.

CARRILlO, R., R. GODOY \& H. PEREDO. 1992. Simbiosis micorrízicas en comunidades boscosas del Valle Central en el sur de Chile. Bosque 13: 57-67.

CAVALLERO, L. 2012. Heterogeneidad ambiental y dispersión de semillas en comunidades de distinta edad post-fuego del noroeste de Patagonia (Tesis Doctoral). Centro Regional Universitario Bariloche. Universidad Nacional del Comahue, S.C. de Bariloche.

CAVIERES, L.A., P. CHACON, A. PENALOZA, M. MOLINA-MONTENEGRO \& M.T.K. ARROYO. 2007. Leaf litter of Kageneckia angustifolia D. Don (Rosaceae) inhibits seed germination in sclerophyllous montane woodlands of central Chile. Plant Ecol. 190: $13-22$.

CAVIERES, L.A., C.L. QUIROZ \& M.A. MOLINAMONTENEGRO. 2008. Facilitation of the nonnative Taraxacum officinale by native nurse cushion species in the high Andes of central Chile: are there differences between nurses? Funct. Ecol. 22: 148-156. COLEY, P.D., J.P. BRYANT \& F.S. CHAPIN. 1985.
Resource availability and plant antiherbivore defense. Sci. Sports 230: 895-899.

COLMET-DAAGE, F., M.L. LANCIOTTI \& A. MARCOLIN. 1995. Importancia de los suelos volcánicos de la Patagonia Norte y Central. INTASAGyP, Centro Regional Patagonia Norte, EEA Bariloche, Área Recursos Naturales.

COLMET-DAAGE, F., M.J. MAZZARINO \& M.L. LANCIOTTI. 1993. Características de los suelos volcánicos en el SO de Chubut. XIV Congreso Argentino de la Ciencia del Suelo, pp. 417-418, Mendoza, Argentina.

CONOVER, W.J. 1980. Practical nonparametric statistics. John Wiley \& Sons, New York.

CORNELISSEN, J.H.C., L. S., E. GARNIER, S. DIAZ, N. BUCHMANN, D.E. GURVICH, P.B. REICH, H. TER STEEGE, M. H.D., M.G.A. VAN DER HEIJDEN, J.G. PAUSAS \& H. POORTER. 2003. A handbook of protocols for standardized and easy measurement of plant functional traits worldwide. Aust. J. Bot. 51: 335-380.

CHOUNDHURY, D. 1988. Herbivore induced changes in leaf-litter resource quality: a neglected aspect of herbivory in ecosystem nutrient dynamics. Oikos 51: 389-393.

DEL VALLE-ARANGO, J.I. 2003. Cantidad, Calidad y nutrientes reciclados por la hojarasca fina en bosques pantanosos del Pacífico Sur colombiano INCI 28: 443-449.

DIEHL, P., M.J. MAZZARINO, F. FUNES, S. FONTENLA, M.E. GOBBI \& J. FERRARI. 2003. Nutrient conservation strategies in native AndeanPatagonian forests. J. Veg. Sci. 14: 63-70.

DOS SANTOS, S.L. \& I.F.M. VALIO. 2002. Litter accumulation and its effect on seedling recruitment in a Southeast Brazilian Tropical Forest. Rev. Bras. Bot. 25: 89-92.

FACELLI, J.M. 1994. Multiple Indirect Effects of Plant Litter Affect the Establishment of Woody Seedlings in Old Fields. Ecology 75: 1727-1735.

FACELLI, J.M. \& E. FACELLI. 1993. Interactions after death: plant litter controls priority effects in a successional plant community. Oecologia 95: 277 282.

FACELLI, J.M. \& S.T.A. PICKETT. 1991a. Indirect effects of litter on woody seedlings subject to herb competition. Oikos 62: 129-128.

FACELLI, J.M. \& S.T.A. PICKETT. 1991b. Plant litter: Its dynamics and effects on plant community structure. Bot. Rev. 57: 1-32.

FACELLI, J.M. \& S.T.A. PICKETT. 1991c. Plant litter: light interception and effects on an old-field plant community. Ecology 72: 1024-1031.

FACELLI, J.M., R. WILLIAMS, S. FRICKER \& B. LADD. 1999. Establishment and growth of 
seedlings of Eucalyptus obliqua: Interactive effects of litter, water, and pathogens. Aust. J. Ecol. 24: 484-494.

FENNY, P. 1970. Seasonal changes in oak leaf tannins and nutrients as a cause of spring feeding by winter moth caterpillars'. Ecology 51: 565-581.

FIORETTO, A., S. PAPA \& A. FUGGI. 2003. Litter-fall and litter decomposition in a low Mediterranean shrubland. Biol. Fertil. Soils 39: 37-44.

FONTENLA, S., R. GODOY, P. ROSSO \& M. HAVRYLENKO. 1998. Root associations in Austrocedrus forests and seasonal dynamics of arbuscular mycorrhizas. Mycorrhiza 8: 29-33.

GOBBI, M.E. 2000. Aspectos ecológicos del manejo productivo de los bosques puros y densos de Austrocedrus chilensis. (Tesis Doctoral). Centro Regional Universitario Bariloche. Universidad Nacional del Comahue, S.C. de Bariloche.

GRAY, J.T. 1982. Community structure and productivity in Ceanothus chaparral and coastal sage scrub of Southern California. Ecol. Monogr. 52: 415-435.

GUTIÉRREZ, J.R. \& F.A. SQUEO 2004. Importancia de los arbustos en los ecosistemas semiáridos de Chile. Ecosistemas 13: 36-45.

HANSEN, R.A. \& D.C. COLEMAN. 1998. Litter complexity and composition are determinants of the diversity and species composition of oribatid mites (Acari: Oribatida) in litterbags. Appl. Soil Ecol. 9: 17-23.

KAVVADIAS, V.A., D. ALIFRAGIS, A. TSIONTSIS, G. BROFAS \& G. STAMATELOS. 2001. Litterfall, litter accumulation and litter decomposition rates in four forest ecosystems in northern Greece. For. Ecol. Manag. 144: 113-127.

KITTREDGE, J., JR. 1939. The annual accumulation and creep of litter and other surface materials in the chaparral of the San Gabriel Mountains, California J. Agric. Res. 58: 537-541.

KITZBERGER, T., E. RAFFAELE, K. HEINEMANN \& M.J. MAZZARINO. 2005. Effects of fire severity in a north Patagonian subalpine forest. J. Veg. Sci. 16: 5-12.

KITZBERGER, T., D.F. STEINAKER \& T.T. VEBLEN. 2000. Effects of climatic variability on facilitation of tree establishment in Northern Patagonia. Ecology 81: 1914-1924.

LAMBERS, H., F.S.I. CHAPIN, F.S. CHAPIN \& T.L. PONS. 2008. Plant physiological ecology. Springer, New York.

LARCHER, W. 2001. Physiological Plant Ecology. Ecophysiology and Stress Physiology of Functional Groups. Springer-Verlag Berlin Heidelberg, New York.

LUSK, C.H., C. DONOSO, M. JIMÉNEZ, C. MOYA, G. OYARCE, R. REINOSO, A. SALDAÑA, P.
VILLEGAS \& F. MATUS. 2001. Descomposición de hojarasca de Pinus radiata y tres especies arbóreas nativas. Rev. Chil. Hist. Nat. 74: 705-710.

MOSTACEDO, B. \& T. FREDERICKSEN. 2000. Manual de métodos básicos de muestreo y análisis en ecología vegetal. Proyecto de Manejo Forestal Sostenible (BOLFOR), Santa Cruz, Bolivia.

NUÑEZ, C., E. RAFFAELE \& M. NÚÑEZ. 2009. When do nurse plants stop nursing?: Temporal changes in water stress levels of the native conifer Austrocedrus chilensis growing within and outside nurse shrubs, in northern Patagonia, Argentina. J. Veg. Sci. 20: $1064-1071$.

OLSON, J.S. 1963. Energy storage and balance of producers and decomposers in ecological systems. Ecology 44: 322-331.

PARITSIS, J., E. RAFFAELE \& T.T. VEBLEN. 2006. Vegetation disturbance by fire affects plant reproductive phenology in a shrubland community in northwestern Patagonia, Argentina. New Zeal. J. Ecol. 30: 387-395.

PAUSAS, J.G. 1997. Litter fall and litter decomposition in Pinus sylvestris forests of the Eastern Pyrenees. $J$. Veg. Sci. 8: 643-650.

PÉREZ-HARGUINDEGUY, N., S. DÍAZ, J. CORNELISSEN, F. VENDRAMINI, M. CABIDO \& A. CASTELLANOS. 2000. Chemistry and toughness predict leaf litter decomposition rates over a wide spectrum of functional types and taxa in central Argentina. Plant Soil 218: 21-30.

PÉREZ, C.A., J.F. GOYA, F. BIANCHINI, J.L. FRANGI \& R. FERNÁNDEZ. 2006. Productividad aérea y ciclo de nutrientes en plantaciones de Pinus taeda L. en el norte de la provincia de misiones, Argentina / Aboveground productivity and nutrient cycle in Pinus taeda L. plantations in the north of the Misiones province, Argentina. Interciencia 31: 794-801.

PÉREZ HARGUINDEGUY, N., C. BLUNDO, D. GURVICH, S. DÍAZ \& E. CUEVAS. 2008. More than the sum of its parts? Assessing litter heterogeneity effects on the decomposition of litter mixtures through leaf chemistry. Plant Soil 303: 151-159.

QUINN, G.P. \& M.J. KEOUGH. 2002. Experimental design and data analysis for biologists. Cambridge University Press, Cambridge.

RAFFAELE, E. \& T.T. VEBLEN. 1998. Facilitation by nurse shrubs of resprouting behaviour in a post-fire shrubland in northern Patagonia, Argentina. J. Veg. Sci. 9: 1-6.

RAFFAELE, E. \& T.T. VEBLEN. 2001. Effects of cattle grazing on early postfire regeneration of matorral in Northwest Patagonia, Argentina. Nat. Areas J. 21: 243-249. 


\section{M. del Paz et al. - Mantillo de especies leñosas de matorrales de la Patagonia}

RHOADES, D.F. \& R.G. CATES. 1976. Towards a general theory of plant antiherbivores chemistry. Recent advances in phytochemestry 10: 168-213.

ROMANYÀ, J., J. CORTINA, P. FALLON, K. COLLEMAN \& P. SMITH. 2000. Modeling changes in soil organic matter after planting fast-growing Pinus radiata on Mediterranean agricultural soils. Eur. J. Soil Sci. 51: 627-641.

SCARFF, F.R. \& M. WESTOBY. 2006. Leaf litter flammability in some semi arid Australian woodlands. Funct. Ecol. 20: 745-752.

SHENGLI, G., M. YUHONG, C. SHENGGUO \& S. WENYI. 2009. Effects of artificial and natural vegetations on litter production and soil organic carbon change in loess hilly areas. Scientia Silvae Sinicae 45: 14-18.

VARELA, S.A., M.E. GOBBI \& F. LAOS. 2006. Banco de semillas de un bosque quemado de Nothofagus pumilio: efecto de la aplicación de compost de biosólidos. Ecol. Austral. 16: 63-78.

VEBLEN, T., T. KITZBERGER, E. RAFFAELE \& D. LORENZ. 2003. Fire History and Vegetation Changes in Northern Patagonia, Argentina. In: VEBLEN, T.T. ,BAKER, W.L., MONTENEGRO G. \& T.W. SWETNAM (eds.), Fire and Climatic Change in Temperate Ecosystems of the Western Americas, pp. 265-295. Springer, New York.

VEBLEN, T.T., A. HOLZ, J. PARITSIS, E. RAFFAELE, T. KITZBERGER \& M. BLACKHALL. 2011. Adapting to global environmental change in Patagonia: What role for disturbance ecology. Ecol. Austral 36: 891-903.

VITOUSEK, P.M. 1982. Nutrient cycling and nutrient use efficiency. Am. Nat. 119: 553-572.

VITOUSEK, P.M. \& J.R. SANFORD. 1986. Nutrient cycling in tropical forests. Annu. Rev. Ecol. Syst. 17: 137-167.

VIVANCO, L. 2008. Efectos de la identidad y diversidad de especies de plantas sobre el reciclado de carbono y nutrientes en bosques templados en Patagonia, Argentina. UBA, Buenos Aires.

VIVANCO, L. \& A. AUSTIN. 2006. Intrinsic effects of species on leaf litter and root decomposition: a comparison of temperate grasses from North and South America. Oecologia 150: 97-107.

VIVANCO, L. \& A.T. AUSTIN. 2008. Tree species identity alters forest litter decomposition through long-term plant and soil interactions in Patagonia, Argentina. J. Ecol. 96: 727-736.

WRIGHT, I.J., P.B. REICH, M. WESTOBY, D.D. ACKERLY, Z. BARUCH, F. BONGERS, J. CAVENDER-BARES, T. CHAPIN, J.H.C. CORNELISSEN, M. DIEMER, J. FLEXAS, E. GARNIER, P.K. GROOM, J. GULIAS, K. HIKOSAKA, B.B. LAMONT, T. LEE, W. LEE, C. LUSK, J.J. MIDGLEY, M.-L. NAVAS, U. NIINEMETS, J. OLEKSYN, N. OSADA, H. POORTER, P. POOT, L. PRIOR, V.I. PYANKOV, C. ROUMET, S.C. THOMAS, M.G. TJOELKER, E.J. VENEKLAAS \& R. VILLAR. 2004. The worldwide leaf economics spectrum. Nature 428: 821-827.

XIONG, S., C. NILSSON \& M. JOHANSSON, E. 2001. Effects of litter accumulation on riparian vegetation: Importance of particle size. J. Veg. Sci. 12: 231-236.

ZAR, J.H. 1996. Biostatistical Analysis Prentice- Hall, Inc., New Jersey.

Recibido el 15 de octubre de 2012, aceptado el 25 de febrero de 2013. 
